\title{
Polarization conversion with a photonic crystal slab
}

\author{
Marine Laroche \\ marine.laroche@institutoptique.fr \\ François Marquier \\ Cédric Vandenbem \\ Jean-Jacques Greffet
}

\begin{abstract}
Laboratoire Charles Fabry de l'Institut d'Optique, CNRS, Université Paris-Sud, Campus Polytechnique, RD128, 91127 Palaiseau, France

Laboratoire Charles Fabry de 1'Institut d'Optique, CNRS, Université Paris-Sud, Campus Polytechnique, RD128, 91127 Palaiseau, France

Laboratoire d'Optique Physique, LPEM UPR5 CNRS, Ecole Supérieure de Physique et Chimie Industrielles, 10, rue Vauquelin, 75005 Paris, France.

Laboratoire de Physique du Solide, University of Namur (FUNDP), 61 rue de Bruxelles, 5000 Namur, Belgium.

Laboratoire Charles Fabry de l'Institut d'Optique, CNRS, Université Paris-Sud, Campus Polytechnique, RD128, 91127 Palaiseau, France
\end{abstract}

We show that a photonic crystal slab can enable efficient polarization conversion. Two mechanisms are identified. The first mechanism relies on the anisotropy of the bulk properties of the metamaterial and is mediated by interferences. The second mechanism is due to the resonant excitation of leaky surface waves at the interface of the photonic crystal. The latter is analogous to the polarization conversion by excitation of surface plasmons on a metallic grating. This is another example of the possibility of mimicking plasmonics with photonic crystals. [DOI: 10.2971/jeos.2008.08038]

Keywords: photonic crystals, surface waves, polarization

\section{INTRODUCTION}

Metallic gratings have been extensively studied for their ability to rotate the plane of polarization of a normally incident linearly polarized beam by $90^{\circ}$. Two mechanisms were demonstrated [1] : the resonant excitation of surface plasmon polaritons (SPPs) [2] and the interferences between the optical fields in the directions parallel and perpendicular to the grating lines caused by the anisotropy introduced by the grating [3].

A photonic crystal slab has several features in common with a metallic grating. It is highly reflective for wavelengths lying in the bandgap and exhibits anisotropy. Also, depending on the truncation of its interface, a photonic crystal slab can support leaky surface waves. Firstly demonstrated for a 1D photonic crystal or multilayer system [4], it has later been shown both theoretically and experimentally for 2D photonic crystals $[5,6]$. Several phenomena known in plasmonics have been transposed to photonic crystals. The beaming of light [7] mediated by surface waves on photonic crystals has been demonstrated. Other phenomena like resonant transmission [8] and coherent thermal emission by excitation of surface waves on a photonic crystal has been demonstrated in [9]. Recently, other nanostructured materials have been studied for their properties of polarization conversion : photonic crystal waveguides [10] and subwavelength gratings illuminated through a glass substrate [11].

In this paper, we show that a photonic crystal slab enables efficient polarization conversion in reflection. We report two mechanisms. The first mechanism is mediated by interferences and is due to the anisotropy of the bulk properties of the metamaterial. The other mechanism is due to the resonant excitation of leaky surface waves at the interface of the photonic crystal.

\section{GEOMETRY AND METHOD}

Using numerical simulations based on Rigourous Coupled Wave Analysis (RCWA) [12], we study the reflection properties of a 2D photonic crystal slab made of germanium (Ge) for wavelengths around $1.55 \mu \mathrm{m}$. In this range of wavelengths, the dielectric constant of $\mathrm{Ge}$ is approximately $\epsilon_{\mathrm{Ge}} \simeq 18.27$ but both dispersion and absorption are taken into account in the calculations using the experimental data from [13]. The geometry of the system is described in Figure 1. It consists in a chessboard of infinitely long square rods, with period $a=0.95 \mu \mathrm{m}$, height $h=0.475 \mu \mathrm{m}$. The thickness of the first layer $h_{\text {term }}$ can take different values. The reflection properties of 9-layer photonic crystal slab are studied in conical diffraction. This means that the photonic crystal is illuminated with a wavevector lying in any plane of incidence, marked by angle $\phi$, and any incident angle $\theta$ as seen in Figure 1. The polarization of the field is defined from the plane of incidence with angle $\Psi=0^{\circ}$ for p-polarization and $\Psi=90^{\circ}$ for s-polarization. 


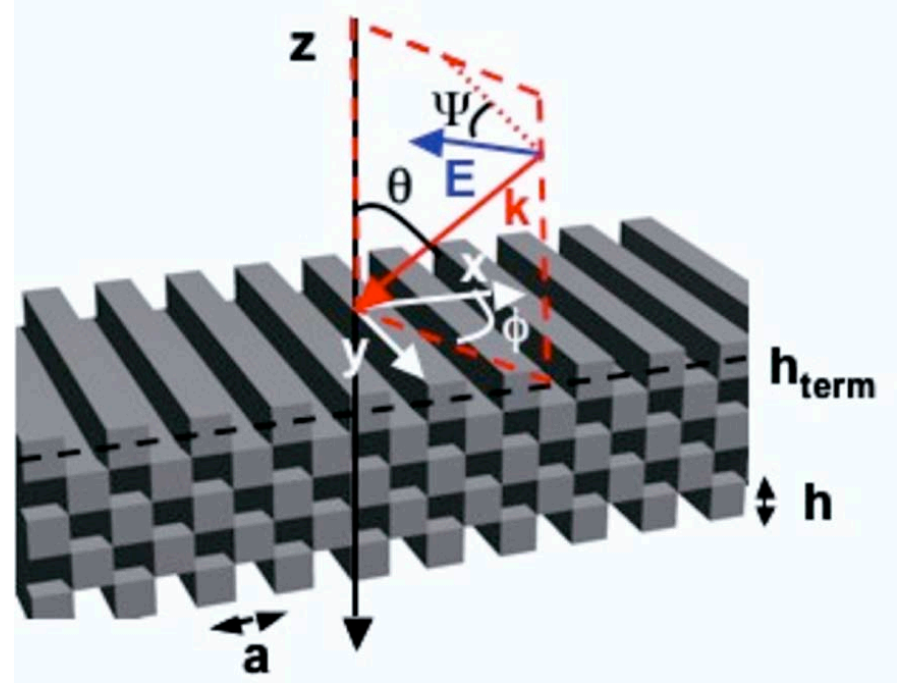

FIG. 12 D photonic crystal made of Ge with period $a=0.95 \mu \mathrm{m}$, height $h=0.475 \mu \mathrm{m}$. $\theta$ is the angle between the incident wavevector and the normal to the interface. $\phi$ is the angle between the $x$-axis and plane of incidence. $p$-polarization is defined by $\Psi=0^{\circ}$, s-polarization by $\Psi=90^{\circ}$. The height of the first layer is denoted $h_{\text {term }}$.
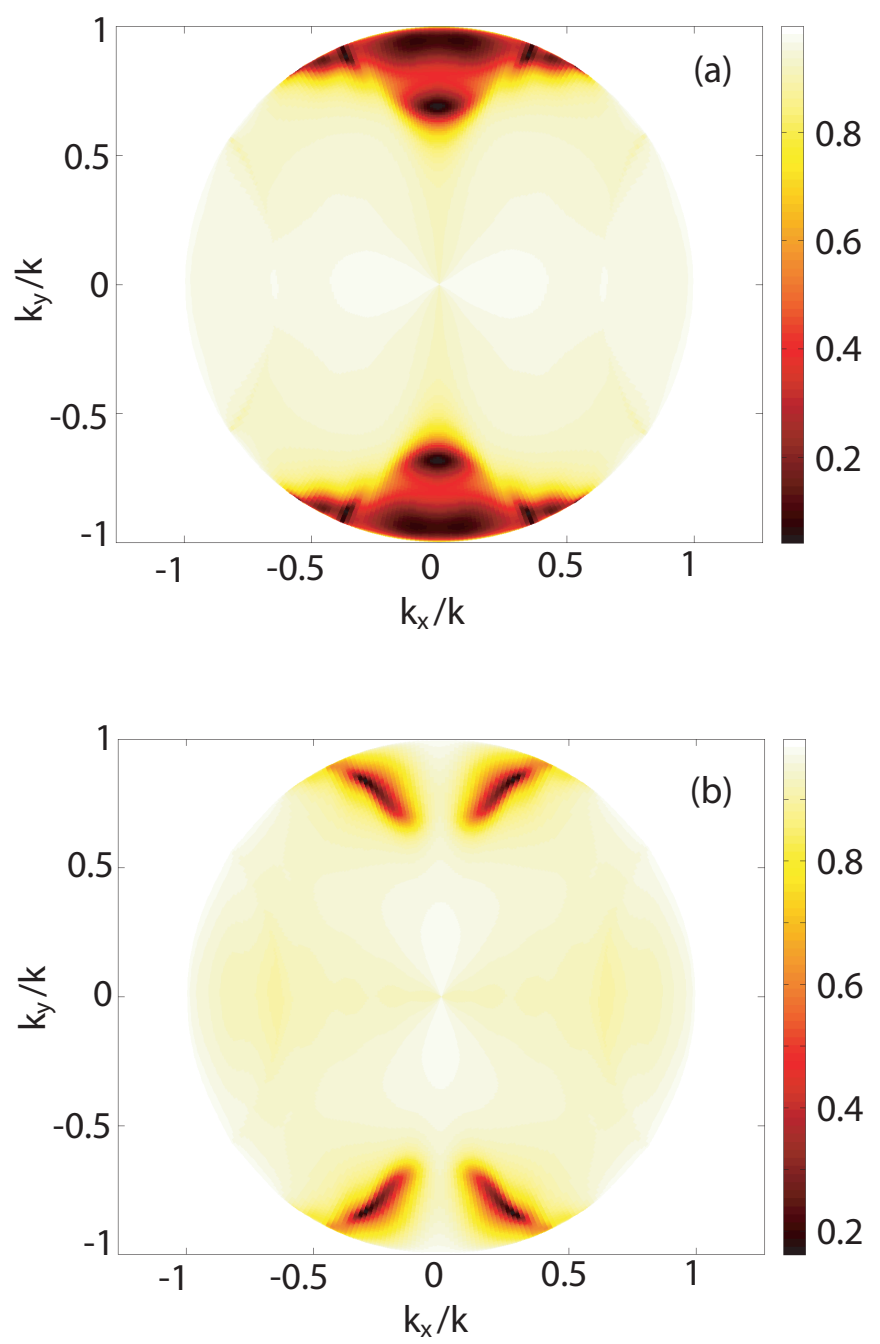

FIG. 2 Polar representation of the total reflectivity at $\lambda=1.55 \mu \mathrm{m}$ for the system described in Figure 1. (a) under p-polarized illumination (b) under s-polarized illumination.
From Figure 2, it can be seen that this structure exhibits a bandgap for both s and p-polarization at $\lambda=1.55 \mu \mathrm{m}$ as the total reflection factor goes beyond $95 \%$ (bright color) for almost all planes of incidence and incident angles.

\section{INTERFERENCE-MEDIATED REGIME}

Let us now investigate the polarization properties of the reflection of such a structure for $\lambda=1.55 \mu \mathrm{m}$ when illuminated by p-polarized incident light. Figure 3(a) displays the part of the incident energy reflected in s-polarization $R_{p s}$ when the photonic crystal is not truncated $\left(h_{\text {term }}=0.475 \mu \mathrm{m}\right)$. On the $x$-axis is the normalized projection of the incident wavevector $\mathbf{k}_{x}=\sin (\theta) \cos (\phi)$ and on the $y$-axis is the normalized projection of the incident wavevector $\mathbf{k}_{y}=\sin (\theta) \sin (\phi)$ so that each point represents a direction of incidence. We focus on the region of the plane where only the zero order is propagating. The zones where other diffracted orders become propagating appear in black colour.

We see that the polarization conversion is highly effective as
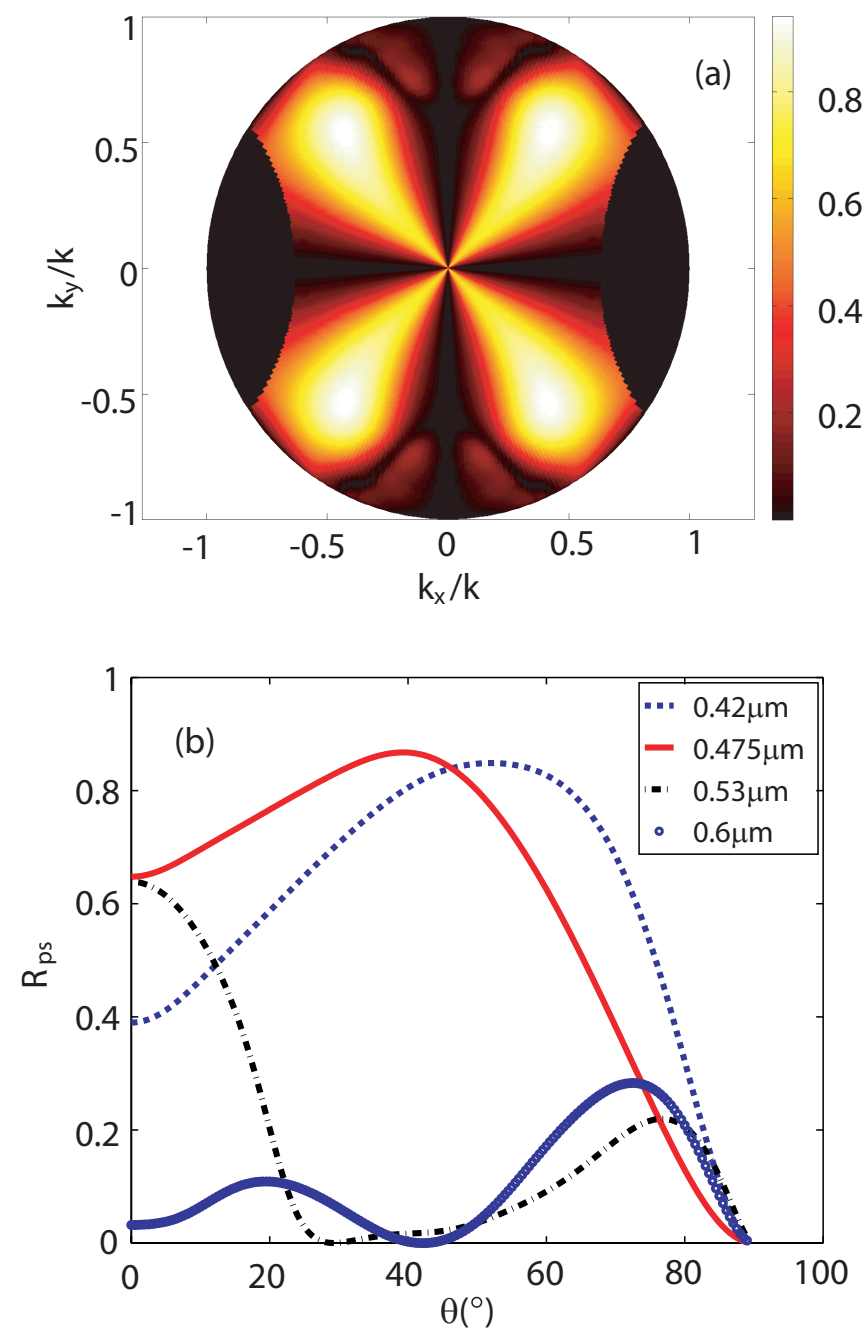

FIG. 3 Interference mediated regime, $\lambda=1.55 \mu \mathrm{m}$ : (a) Polar representation of the reflectivity under $\mathrm{p}$-polarized illumination into s-polarization $R_{p s}$ for an untruncated photonic crystal slab (with $h_{\text {term }}=0.475 \mu \mathrm{m}$ ). (b) $R_{p s}$ versus the incident angle $\theta$ for $\phi=45^{\circ}$ and for several termination heights $h_{\text {term }}$. 
$R_{p s}$ reaches $84 \%$ around $\phi=45^{\circ}$ and for a broad range of incident angles around this value. This polarization conversion is easy to interpret. As in the case of a metallic grating, the 1D periodic structure of the photonic crystal introduces anisotropy. The components of the electric field parallel $E_{\|}$and perpendicular $E_{\perp}$ to the axis of the square rods, experience a different dephasing when reflected at the interface. When incident around $\phi=45^{\circ}$, both components are equal so that a dephasing of $\pi$ between the two components will lead to total polarization conversion. Depending on the height of the first layer, as shown in Figure 3(b), the dephasing and consequently the polarization conversion can be tuned. Those results do not depend on the number of layers as long as this number is large enough to create the bandgap.

\section{SURFACE WAVE-MEDIATED REGIME}

For smaller values of the height of the first layer, the reflection pattern $R_{p s}$ is radically different. This is illustrated in Figure 4(a) where the reflection pattern $R_{p s}$ is displayed in polar coordinates. The region where the polarization conversion is larger than $50 \%$ follows a sharp circular line. From quasiisotropic, the polarization conversion has become highly directional. This is illustrated in Figure $4(\mathrm{~b})$ where $R_{p s}$ is plotted versus the incident angle for a given plane of incidence (for $\phi=33^{\circ}$ ) and several values of the height of the interface layer. We observe that the height modifies both the angle of resonance and the efficiency of the polarization conversion.

The reflected electric field in vacuum can be written with the Rayleigh expansion:

$$
E_{x}^{r}(x, y, z)=\sum_{p} E_{x}^{(p)} e^{i\left(k_{x}+p \frac{2 \pi}{a}\right) x} e^{i\left(k_{y} y+\gamma_{p} z\right)}
$$

where $E_{x}^{r}$ is the $x$-component of the reflected field, $k_{x}$ the incident wavevector component in the $x$-direction, $k_{y}$ in the $y$ direction and $\gamma_{p}^{2}=\frac{\omega^{2}}{c^{2}}-\left(k_{x}+p \frac{2 \pi}{a}\right)^{2}-\left(k_{y}\right)^{2}$ with $\Im\left(\gamma_{p}\right)>0$ its component in the $z$-direction. Figure 5 represents the amplitude of the -1 evanescent order (normalized to the amplitude of the incident field) of the $x$-component of the reflected field when $h_{\text {term }}=0.175 \mu \mathrm{m}$. We can see an enhancement of the amplitude by a factor of 3 which coincides with the peak of polarization conversion. Nevertheless, we can notice that the enhancement of the -1 evanescent order also exists near $k_{y}=0$, but there is no polarization conversion for symmetry reason. In this case, the enhancement yields an increase of the absorption as already shown in [9]. The amplitudes of the other evanescent orders (not shown here) do not exhibit similar enhancement. Hence, the field at the peak of polarization conversion has the structure of a leaky surface waves with a resonant evanescent order and a 0 propagating order. Here, the leaky surface wave behaves similarly to surface phonon polaritons on lamellar gratings [14] as it can be excited in both $\mathrm{s}$ and p polarization when the plane of incidence is strictly between $\phi=0^{\circ}$ and $\phi=90^{\circ}$. By reciprocity, it implies that it can decouple back in s and p-polarization leading to polarization conversion. This physical mechanism is very similar to the polarization conversion through the excitation of surface plasmons [2]. The resonant behaviour of the polarization conversion is due to the excitation of surface waves that can be
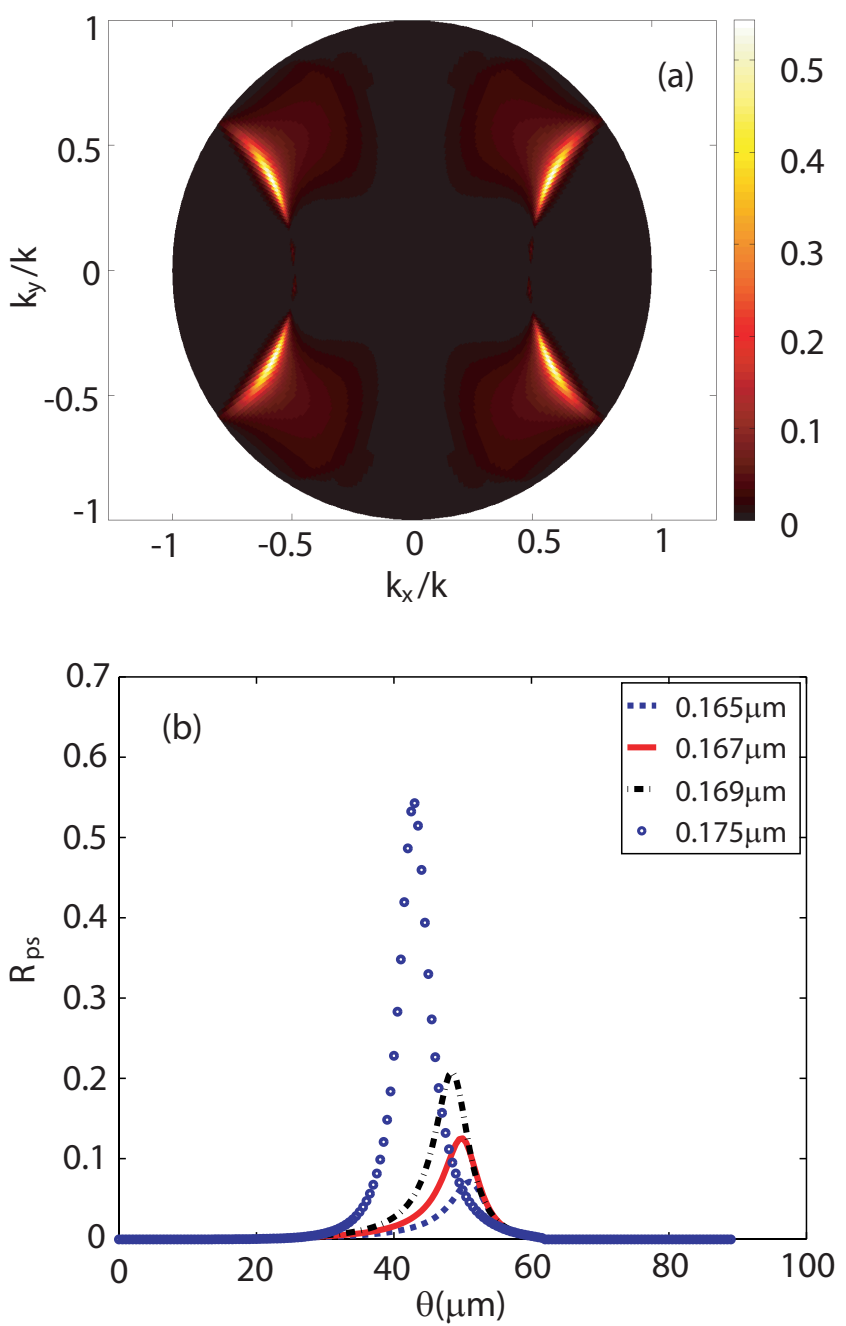

FIG. 4 Surface-wave mediated regime, $\lambda=1.55 \mu \mathrm{m}$ : (a) polar representation of the reflectivity under $p$-polarized illumination into s-polarization $R_{p s}$ for truncated photonic crystal slab with $h_{\text {term }}=0.175 \mu \mathrm{m}$. (b) $R_{p s}$ versus the incident angle $\theta$ for $\phi=33^{\circ}$ and for several termination heights $h_{\text {term }}$.

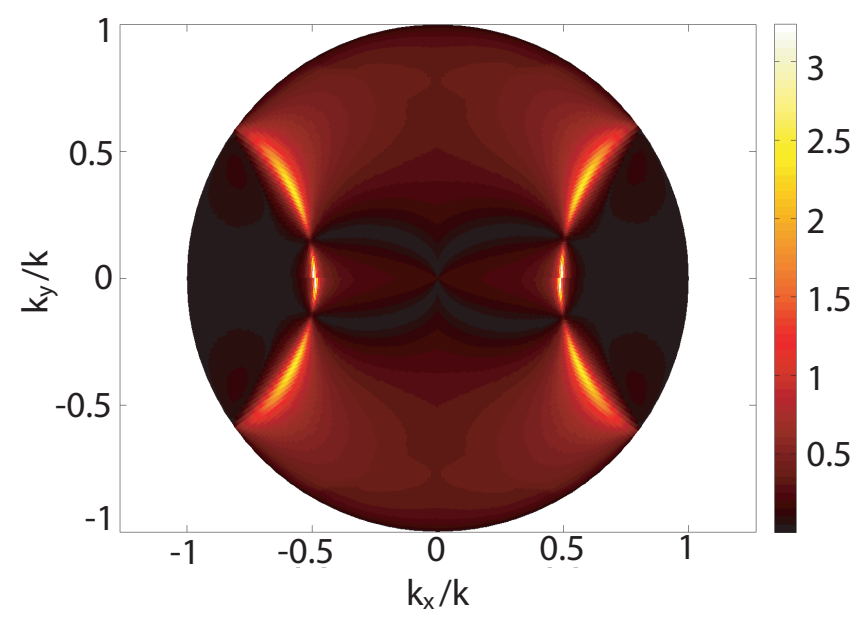

FIG. 5 Surface-wave mediated regime : Amplitude of the -1 evanescent order of the $x$-component of the electric field $E_{x}^{(-1)}$ (normalized to the amplitude of the incident field) when $h_{\text {term }}=0.175 \mu \mathrm{m}$.

excited for suitable terminations of the photonic crystal. Also, Figure 4(b) illustrates the fact that the truncation modifies the 
dispersion relation of the surface waves as already discussed in $[8,9]$.

\section{CONCLUSION}

To conclude, we have shown that a photonic crystal slab enables efficient polarization conversion. Also, it is possible to switch from the interference-mediated regime of polarization conversion to the resonant regime by tuning the termination of the photonic crystal. The resonant regime gives further evidence that a photonic crystal provides an interesting alternative to plasmonics. The advantage of the photonic crystal slab is that it is made with dielectric materials exhibiting lower losses than metals and that the dispersion relation of surface waves can be modified with the lattice parameters.

\section{ACKNOWLEDGEMENTS}

C. V. is a postdoctoral researcher of the Belgian National Fund for Scientific Research (FNRS).

\section{References}

[1] I. R. Hooper, and J. R. Sambles, "Broadband polarizationconverting mirror for the visible region of the spectrum" 0pt. Lett. 27, 2152 (2002).

[2] G.P. Bryan-Brown, J. R. Sambles, and M.C. Hutley, "Polarisation Conversion through the Excitation of Surface Plasmons on a Metallic Grating" J. Mod. Opt. 37, 1227 (1990).

[3] R.A. Watts, and J. R. Sambles, "Reflection gratings as polarization converters" Opt. Commun. 140, 179 (1997).

[4] P. Yeh, A. Yariv, and C-S. Hong, "Electromagnetic propagation in periodic stratified media. I. General theory" J. Opt. Soc. Am. 67, 423 (1977).

[5] R.D. Meade, K.D. Brommer, A.M. Rappe, and J.D. Joannopoulos, "Electromagnetic Bloch waves at the surface of a photonic crystal" Phys. Rev. B 44, 10961 (1991).

[6] W.M. Robertson, G. Arjavalingam, R. D. Meade, K. D. Brommer, A.M. Rappe, and J. D. Joannopoulos, "Observation of surface photons on periodic dielectric arrays" Opt. Lett. 18, 528 (1993).

[7] P. Kramper, M. Agio, C. Soukoulis, A. Birner, F. Muller, R. Wehrspohn, U. Gosele, and V. Sandoghdar, "Highly Directional Emission from Photonic Crystal Waveguides of Subwavelength Width" Phys. Rev. Lett. 92, 113903 (2004).

[8] M. Laroche, R. Carminati, and J.-J. Greffet, "Resonant optical transmission through a photonic crystal in the forbidden gap" Phys. Rev. B 71, 155113 (2005).

[9] M. Laroche, R. Carminati, and J.-J. Greffet, "Coherent Thermal Antenna Using a Photonic Crystal Slab" Phys. Rev. Lett. 96, 123903 (2006).

[10] A.D. Bristow, V.N. Astratov, R. Shimada, I.S. Culshaw, M.S. Skolnick, D.M. Whittaker, A. Tahrauoi, and T. F. Krauss, "Polarization Conversion in the Reflectivity Properties of Photonic Crystal Waveguides" IEEE J. Quantum Elect. 38, 880 (2002).

[11] N. Passily, K. Ventola, P. Karvinen, P. Laakkonen, J. Turunen, and J. Tervo, "Polarization conversion in conical diffraction by metallic and dielectric subwavelength gratings" Appl. 0pt. 46, 4258 (2007).

[12] N. Chateau, and J.-P. Hugonin, "Algorithm for the rigorous coupledwave analysis of grating diffraction" J. Opt. Soc. Am. A 11, 1321 (1994).

[13] E. D. Palik, Handbook of Optical Constants of Solids (Academic Press Inc., San Diego, 1985)

[14] F. Marquier, C. Arnold, M. Laroche, J.J. Greffet, and Y. Chen, “Degree of polarization of thermal light emitted by gratings supporting surface waves" Opt. Express 16, 5305 (2008). 